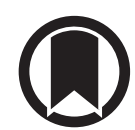

CrossMark

\section{Epidemiological characteristics of SARS-CoV-2 infections in Shaanxi, China by 8 February 2020}

To the Editor:

In December 2019, a new coronavirus named severe acute respiratory syndrome-coronavirus-2 (SARS-CoV-2) causing severe acute respiratory disease emerged in the region of Wuhan, China $[1,2]$. The National Health Commission of the People's Republic of China named pneumonia infected with SARS-CoV-2 as novel coronavirus pneumonia (NCP). Evidence pointed towards person-to-person transmission [3-5]. As of 8 February 2020, China had reported 34673 confirmed cases and more than 27657 suspected cases of NCP across 34 Chinese provinces or municipalities, with 106 fatalities. Most of the studies focused on the epidemic situation in Wuhan, but a few studies focused on provinces outside Wuhan. Shaanxi is located to the northwest of the Hubei Province and is adjacent to it, with a population of 38.64 million. Since the first case of NCP was confirmed in Shaanxi on 23 January 2020, 195 cases have been confirmed, but there was no relevant epidemiological study. In order to better understand the epidemic of the new coronavirus in the Shaanxi Province and provide the basis for the future development of control measures, this article aims to report the epidemiological outcomes of the 195 confirmed cases infected with SARS-CoV-2 and to compare the clinical features between patients with or without a history of exposure in Wuhan.

We reviewed the epidemiological characteristics and outcome date of 195 laboratory confirmed cases of SARS-CoV-2 infection reported by the Health Commission of the Shaanxi Province. The study period of these patients was from 23 January 2020 to 7 February 2020. The investigation data included sex, contact history of Wuhan, date of onset, date of diagnosis, severity of the case and other information. Two researchers independently reviewed the data.

By February 7 2020, 195 cases of NCP were confirmed in the Shaanxi Province, as shown in figure la. There were 88 cases in Xi'an, 14 in XianYang, eight in Yan'An, three in YuLin, 10 in WeiNan, seven in TongChuan, seven in ShangLuo, 23 in HanZhong, 13 in BaoJi and 22 in AnKang. In total, there were 539 suspected cases across all provinces. The main confirmed cases of early stage NCP were patients with SARS-CoV-2 from Wuhan (figure $1 b$ ).

The characteristics of patients with SARS-CoV-2 infection in the Shaanxi province were as follows. The mean age was $44.13 \pm 15.8$ years, $25(12.82 \%)$ cases were 20 years of age, $23(11.79 \%)$ were $20-29$ years of age, $79(40.51 \%)$ were $30-49$ years of age, 28 (14.36\%) were 50-59 years of age, $29(14.87 \%)$ were $60-69$ years of age and $11(5.64 \%)$ cases were aged $>70$ years. The majority of cases were male $(66.15 \%)$. $190(97.44 \%)$ patients were in stable state and five $(2.56 \%)$ patients were severe. The average time from onset to diagnosis was $7.35 \pm 3.73$ days.

According to the analysis of the patients with or without Wuhan exposure history, we found that the average age was $39.79 \pm 15.76$ years for patients with Wuhan exposure and $49.19 \pm 14.44$ years for patients without Wuhan exposure $(\mathrm{p}<0.05)$. The average time from onset to diagnosis was $7.08 \pm 4.04$ days for patients with Wuhan exposure and $7.65 \pm 3.37$ for patients without Wuhan exposure $(\mathrm{p}<0.05)$.

Of 105 patients analysed with Wuhan exposure history, 71 of them had long-term exposure and 18 had short-term exposure (meeting, layover from transport hubs and travel). Most of the infected patients were male (long-term exposure: 90.14\%; short-term exposure: $72.22 \%$ ). The time from onset to diagnosis was

@ERSpublications

Patients confirmed with novel coronavirus pneumonia in Shaanxi Province mainly have a history of Wuhan contact. There are characteristics of family aggregation and concealment. http://bit.ly/2x7xs $1 \mathrm{~V}$

Cite this article as: Yao Y, Tian Y, Zhou J, et al. Epidemiological characteristics of SARS-CoV-2 infections in Shaanxi, China by 8 February 2020. Eur Respir J 2020; 55: 2000310 [https://doi.org/10.1183/ 13993003.00310-2020]. 

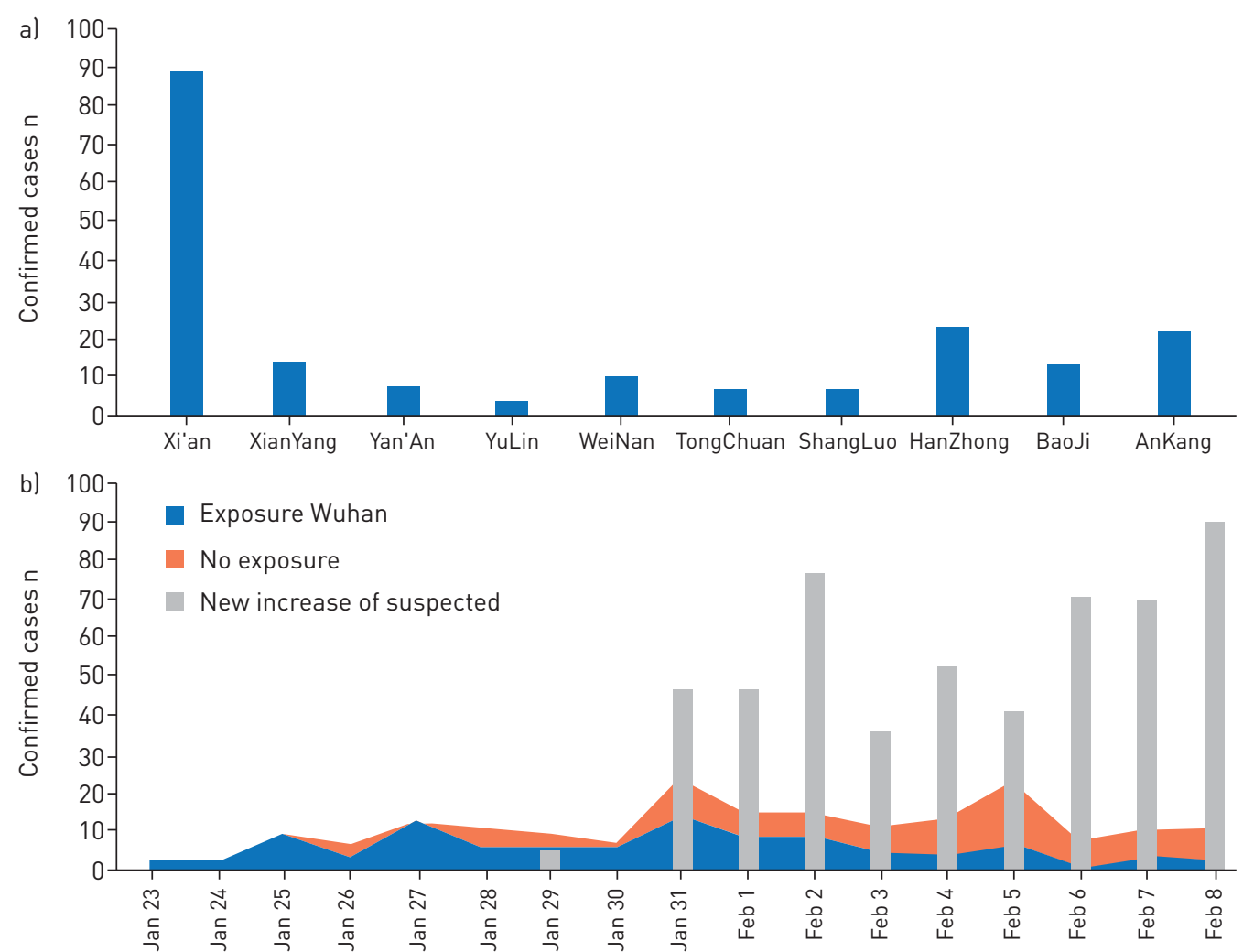

FIGURE 1 Patients with severe acute respiratory syndrome-coronavirus-2 (SARS-CoV-2) infection in Shaanxi province by 8 February 2020. a) The number of confirmed patients with SARS-CoV-2 infection in different cities of the Shaanxi Province. Xi'an has the largest number of confirmed cases and YuLin has the smallest. b) Date of daily confirmed cases of SARS-CoV-2 infection of Wuhan exposure history for the year 2020. The main confirmed cases of early stage are mainly imported cases.

$6.06 \pm 4.85$ days and 7.22 3.75 days for long-term versus short-term exposure, respectively. Of the 195 confirmed cases, 90 had no Wuhan exposure history. We divided these patients into direct contact and indirect contact with people from Wuhan who had returned to Shaanxi. They were mainly middle-aged patients. The average age was $48.52 \pm 15.99$ years and $50.46 \pm 13.38$ years for direct and indirect contact cases, respectively. The male:female ratios were 16:13 and 13:22 for direct and indirect cases, respectively. Furthermore, 26 patients had unknown contact history. Most of the patients were in a stable state but five cases were severe. Up to 7 February 2020, 8.72\% of 195 patients had been discharged. The average age of discharged patients was $34.53 \pm 13.08$ years. The length of hospitalisation was $12.76 \pm 3.42$ days and nine (52.94\%) were male. In addition, there were two family aggregated cases. Case 1 was a 50-year-old male who drove to Wuhan on 13 January 2020 returning to HanZhong on 17 January 2020 and developed symptoms on 18 January 2020. His wife drove to Wuhan with him. They were confirmed with SARS-CoV-2 on the 27 January 2020. Then nine people were infected by them. Case 2, was a family of three living in Wuhan, they drove to Tongchuan city to visit their relatives on 20 January 2020 and four people were infected by them. Up to 7 February 2020, 17 (8.72\%) out of 195 patients had been discharged.

To our knowledge this is the largest case series to date of confirmed patients with SARS-CoV-2 infection. In addition, this is the first study to focus on the SARS-CoV-2 outside Wuhan. We found that the majority of patients were middle-aged males. This result is consistent with the research of GuAN et al. [6] in Wuhan. They found that the median age of patients with SARS-CoV-2 infection was 47 years, and $58.1 \%$ were male. Our findings are also consistent with the study of HUANG et al. [7] who also concluded that the majority of infected patients were male (73\%). We further divided patients with NCP into a Wuhan exposure group and a non-Wuhan exposure group. We found that patients without Wuhan exposure history were older than those with Wuhan exposure history, and the difference was statistically significant. This may be due to the fact that most of the patients with SARS-CoV-2 infection in Shaanxi were imported cases, and most of them were migrant workers in Hubei who returned home during the Spring Festival. In addition, older people are more likely to be infected with SARS-CoV-2. Furthermore, the day from onset to diagnosis for patients with Wuhan exposure history was longer than those without 
Wuhan exposure history. This is easy to understand, because most of the patients without Wuhan exposure were infected by imported patients. The Wuhan exposure group was further divided into a long-term and a short-term exposure group. In conclusion, the male patients with SARS-CoV-2 accounted for a large proportion of cases and the day from onset to diagnosis was $\sim 7.35$ days. In addition, family clustering cases were found in Shaanxi Province.

Yang Yao $\oplus^{1,2}$, Yao Tian ${ }^{1,2}$, Jing Zhou ${ }^{1}$, Xuan $\mathrm{Ma}^{1}$, Min Yang ${ }^{1}$ and ShengYu Wang ${ }^{1}$

${ }^{1}$ Dept of Pulmonary and Critical Care Medicine, The Xi'an Medical University Affiliated Hospital, Xi'an, Shaanxi, People's Republic of China. ${ }^{2}$ Both authors contributed equally to this work.

Correspondence: ShengYu Wang, Dept of Pulmonary and Critical Care Medicine, The First Affiliated Hospital of Xi'an Medical University, Xi’an, 710077, Shaanxi, People’s Republic of China. E-mail: wangshengyu@yeah.net

Received: 10 Feb 2020 | Accepted after revision: 22 Feb 2020

Author contributions: Y. Yao and Y. Tian designed the study, conducted analysis, and drafted the work; J. Zhou made substantial contribution to design of the work, interpretation of the work, and revising the draft for important intellectual content; X. Ma made substantial contribution to analysis and interpretation of the work; M. Yang helped with access to the data, data management, and analysis; S.Y. Wang made substantial contributions to the conception of the work, revising the draft for important intellectual content, and agreed to be accountable for all aspects of the work in ensuring that questions related to the accuracy or integrity of any part of the work are appropriately investigated and resolved.

Conflict of interest: None declared.

\section{References}

1 Zhu N, Zhang D, Wang W, et al. A novel coronavirus from patients with pneumonia in China. N Engl J Med 2020; 382: $727-733$.

2 Chan JF, Yuan S, Kok KH, et al. A familial cluster of pneumonia associated with the 2019 novel coronavirus indicating person-to-person transmission: a study of a family cluster. Lancet 2020; 395: 514-523.

3 Chen J. Pathogenicity and transmissibility of 2019-nCoV - a quick overview and comparison with other emerging viruses. Microbes Infect 2020: 22: 69-71.

4 Richardson P, Griffin I, Tucker C, et al. Baricitinib as potential treatment for 2019-nCoV acute respiratory disease. Lancet 2020; 395: e30-e31.

5 Wang DW, Hu B, Chang H, et al. Clinical characteristics of 138 hospitalized patients with 2019 novel coronavirus-infected pneumonia in Wuhan, China. JAMA 2020; 323: 1061-1069.

6 Guan WJ, Ni ZY, Hu Y, et al. Clinical characteristics of coronavirus disease 2019 in China. N Engl J Med 2020; in press [https://10.1056/NEJMoa2002032].

7 Huang C, Wang Y, Li X, et al. Clinical features of patients infected with 2019 novel coronavirus in Wuhan, China. Lancet 2020; 395: 497-506. 